\title{
Burkhard Reber, Genf (1848-1926), und sein Beitrag zur Geschichte der Medizin und Pharmazie
}

Von Paul Röthlisberger

Burkhard Reber lebte während fast eines halben Jahrhunderts in Genf. 1879 wurde er Chefapotheker am Kantonsspital. Da es dem Unterricht und der Forschung diente, stand er mit den Mitgliedern der 1876 eröffneten Medizinischen Fakultät in Verbindung. Auch zeichnete er sich in vielseitiger Weise im wissenschaftlichen Leben Genfs aus. Ihn vorzustellen, erscheint uns um so zeitgemäßer, als sich 1976 der 50. Todestag Rebers jährte und noch keine Würdigung und vollständige bibliographische Erfassung seines medizin- und pharmaziehistorischen Schaffens besteht.

\section{Jugend und Studienjahre}

Burkhard Reber wurde am 11. Dezember 1848 als Sohn eines Landwirte-Ehepaares in Benzenschwil im aargauischen Freiamt geboren. Obwohl er wie seine Geschwister schon früh zur Mitarbeit im elterlichen Hof angehalten wurde, zeigte der Knabe Neigung zur Zoologie, zum Sammeln von Versteinerungen und zur Pflege eines kleinen botanischen Gartens. Neben den Eltern gewann sein Onkel Hans, der als Gemeindepräsident wirkte und über eine breite Allgemeinbildung verfügte, durch seine Gespräche mit dem Neffen einen bestimmenden Einfluß. Ob die Tatsache, daß 1860 unter dem jetzigen Schulhaus Gräber mit gut erhaltenen Schädeln und Knochen entdeckt wurden und daß unweit dieser Stelle eine für die Bronzezeit charakteristische Lanze gefunden wurde, das spätere Interesse Rebers an der Archäologie anregte, ist uns nicht bekannt.

Im Geburtsort und auch in Schoren, wohin die Eltern später übersiedelten, war der junge Reber stets der Klassenerste. Dem 13jährigen erklärte der Lehrer, daß er ihm nichts mehr beibringen könne. Da im Schüler stets der brennende Wunsch nach einem höheren Studium lebendig war, erlaubte ihm schließlich die Mutter trotz mancher Hindernisse den Besuch der Bezirksschule in Muri. Auch dort zeigte der Jüngling eindrückliche Kenntnisse in den Naturwissenschaften, so daß ihn der Fachlehrer mit der Neuordnung der mineralogischen und botanischen Sammlung betraute. In Muri machte Reber die Bekanntschaft von Dr. Theodor Simmler, dem Mitgründer des Schweizerischen Alpenclubs, der als Chemiker und Lehrer an der dortigen landwirtschaftlichen Schule wirkte und für den vielseitig aufgeschlossenen jungen Reber väterliche Zuneigung hegte. Mit der Zeit zogen ihn auch die Archäologie und die Geschichte an. So entdeckte er 1866 in 
der Umgebung von Muri die Überreste einer römischen Villa, die in der Folge durch Fachkreise ausgegraben wurde.

Bei der Berufswahl schlug der Schulrektor den Besuch des Seminars vor, damit sich Reber dem Lehrfach zuwenden könne. Dies entsprach jedoch nicht den Vorstellungen des Schülers, der sich den Naturwissenschaften widmen wollte. Deshalb entschloß er sich, Apotheker zu werden, wozu er am 1. Mai 1868 eine Lehre in Weinfelden antrat. Dort stand er in den Sommermonaten meist schon morgens um zwei oder drei Uhr auf, um sich zur Anlage eines Herbariums dem Botanisieren zu widmen, denn schon um $6 \mathrm{Uhr}$ hatte er auf seinem Posten in der Apotheke zu stehen. Die neue Umgebung war reich an Pflanzen und historischen Stätten. Bei den Streifzügen entdeckte er im Torfmoor von Heimenlachen im Thurgau eine Pfahlbausiedlung. Der Fund brachte ihn mit dem bekannten Urgeschichtsforscher Ferdinand Keller in Zürich in Verbindung; diese blieb bis zu dessen Tod bestehen. Die ersten archäologischen Veröffentlichungen Rebers betreffen diese Siedlung und weitere Funde im Kanton Thurgau. Seit dem Antritt seiner Lehre fing er auch an, alte Gegenstände und Dokumente aus der Geschichte der Pharmazie zu sammeln, die damals oft achtlos weggelegt wurden. Im Oktober 1871 trat Reber zur weiteren Ausbildung in eine Apotheke von Zofingen ein, von wo er in der Freizeit zahlreiche botanische und geologische Exkursionen in die nähere und weitere Umgebung, auch in den aargauischen Jura, unternahm. Auf der Staffelegg entdeckte er Coelestin-Geoden $\left(\mathrm{SrSO}_{4}\right)$ und versorgte aus dieser Fundstelle zahlreiche Museen und Liebhaber. 1872-1874 weilte Reber in Neuenburg, wo er zunächst in einer Apotheke tätig war, dann als Student in die Akademie eintrat und sein propädeutisches Examen ablegte. Auch während dieses Welschlandaufenthaltes wurden die vielseitigen Sammeltätigkeiten in der Umgebung fortgesetzt. Er fand zahlreiche Freunde, namentlich, nachdem er in die Studentenverbindung Zofingia eingetreten war. Nach einem Semester in Straßburg führten die Studien Reber an die Universität Zürich, wo er 1877 das Staatsexamen als Apotheker bestand. In der Folge übernahm er Verwalterstellen in Apotheken von Aarau, Baden und Schaffhausen. Für Einzelheiten über den Werdegang Rebers sei auf die kurzen biographischen Darstellungen verwiesen (1897¹, p. XXXIIIff.).*

\section{Berufliche Tätigkeit}

1879 wurde Reber an den neugeschaffenen Posten eines Chefapothekers am Kantonsspital Genf berufen. Das Gehalt entsprach keineswegs der Bedeutung der

* Die Jahrzahl bedeutet das Publikationsjahr, die hochgesetzte kleine Zahl die Reihenfolge der Publikation Rebers im betreffenden Jahr in der Bibliographie, S. 227-231. 

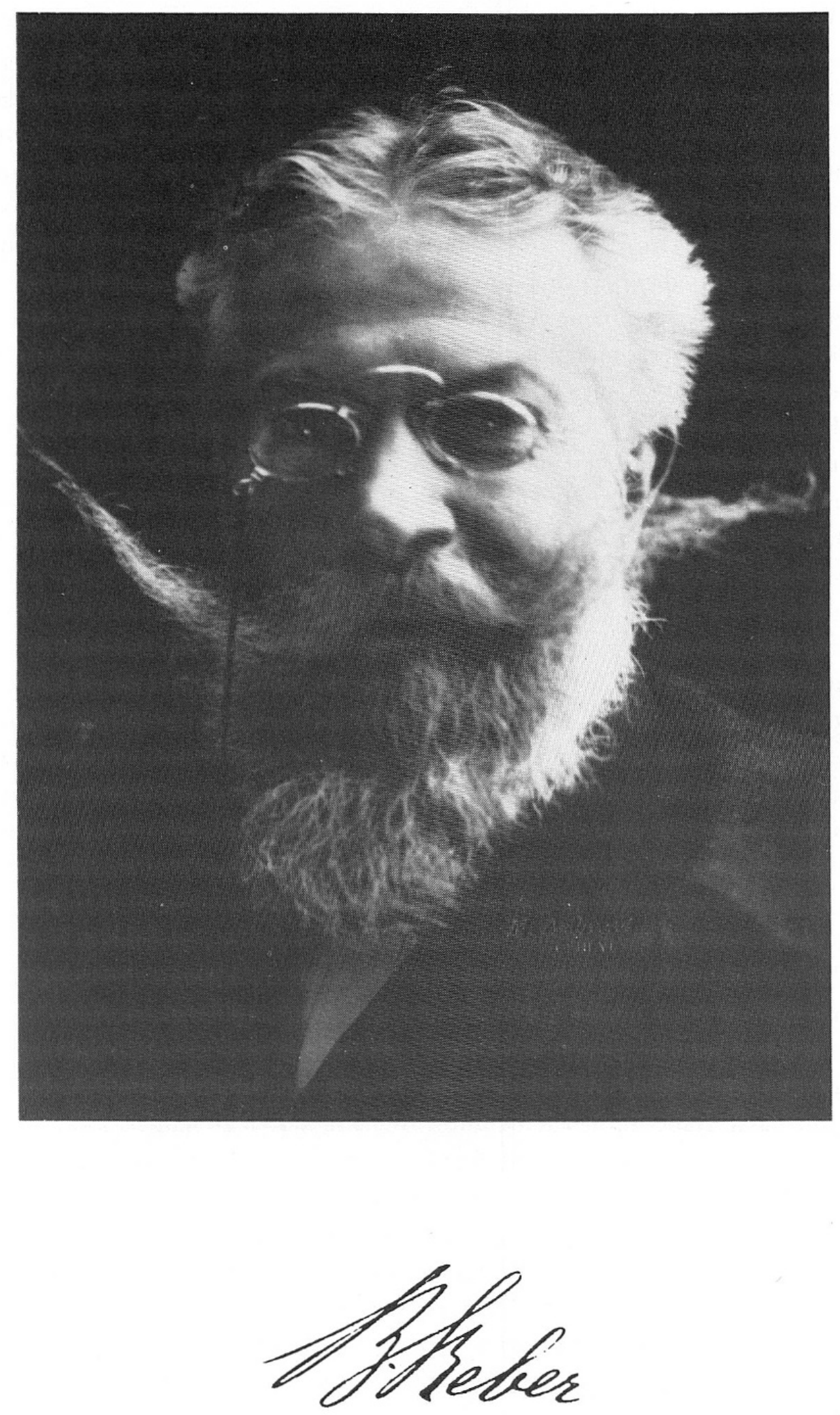
Stellung. Man sicherte jedoch dem Inhaber zu, daß er mit einer Besserstellung rechnen könne, wenn nach Ablauf des Probejahres gegenüber dem bisherigen System, das den einzelnen Kliniken die Freiheit der Arzneimittelbeschaffung in den Apotheken Genfs ließ, sich durch den zentralen Einkauf der Spitaloffizin wesentliche Einsparungen ergäben. Außerdem wurde Reber ein Lehrstuhl an der Universität in Aussicht gestellt.

Mit diesen vielversprechenden Perspektiven nahm Reber die Ernennung an, da ihn eine ausgesprochene Vorliebe für die wissenschaftliche Tätigkeit anzog. Mit Schwung ergriff er seine neue Aufgabe. Doch bald zeigte es sich, daß er eine undankbare Arbeit übernommen hatte. Die Einrichtungen der bisherigen Apotheke befanden sich in einem miserablen Zustand. Zwar sollte die von den Behörden gutgeheißene neue Organisation auch die Einsparung teurer Spezialitäten und die Verhinderung eines unwirtschaftlichen Medikamentenverbrauchs erzielen; doch waren im Spitalbetrieb alte Gewohnheiten zu stark eingewurzelt. Dennoch konnte Reber die früheren Ausgaben für Medikamente um rund 50\% senken. Dies wird durch die nüchternen Tatsachen des Jahresberichtes von 1879 belegt, der einleitend vermerkt, daß neu als Chefapotheker «M. Burckhard Raeber [sic!], Argovien, pharmacien patenté» berufen worden sei. «La pharmacie... a continué à se développer pendant le courant de 1879, à notre entière satisfaction. Malgré les tâtonnements et les difficultés inhérents à toute nouvelle entreprise, les résultats... présentent une réduction notable du coût de la pharmacie et une réelle amélioration dans le fonctionnement de ce service, qui laissait à désirer. Mais le vrai succès a été la régularité obtenue, aussi bien que la bonne qualité des médicaments et la prompte exécution des ordonnances. ${ }^{2}(1879$, p. 7.) Die unbestreitbaren Erfolge waren für die Anhänger der früheren «Ordnung» ein wahrer Donnerschlag - «un véritable coup de massue», bemerkt Reber. Allerdings versuchte man die Neuregelung zu beeinträchtigen, da sie die früheren Mißbräuche und die schamlose Ausbeutung durch die Klüngelwirtschaft und Gefälligkeiten in der Vergangenheit klar bewies («prouvait à l'évidence les abus et l'exploitation éhonté du système de favoritisme et complaisance du passé») ${ }^{1}$ (p.5). Auch im zweiten Amtsjahr hatte Reber immer noch unter Gehässigkeiten zu leiden; zwar verzichtete man darauf, ihn in seiner Stellung zu 'sprengen', wünschte ihn aber zum Kuckuck. Trotz ständiger Unannehmlichkeiten und Schwierigkeiten mit der Verwaltung und der Spitaldirektion, den Oberärzten und Professoren - letztere waren, wie Reber bemerkt, von den Assistenten und dem Pflegepersonal falsch informiert - verlor er seine Ruhe nicht, sondern führte die übernommene Aufgabe unbeirrbar fort. Seine Hauptsorge galt einer gewissenhaften Herstellung der Medikamente und einwandfreien Belieferung der Kliniken. Daneben befaßte er sich 
mit dem Ersatz ausländischer, vor allem teurer Spezialitäten durch Eigenpräparate aus der Spitaloffizin. So gelang ihm die Erfindung des seinerzeit vielverbreiteten 'Terebens', das als Antiseptikum und Desinfektionsmittel diente. Hätte Reber nicht in uneigennütziger Weise dessen Herstellungsverfahren veröffentlicht, so hätte ihm die geschäftliche Verwertung wohl ein Vermögen eingebracht ${ }^{1}$ (p.3). Auch in den Jahresberichten für 1880 und 1881 lauten die Beurteilungen Rebers durchaus schmeichelhaft, so 1880 : «La pharmacie installée il y a deux ans, continue à fonctionner à notre entière satisfaction. Les résultats qu'on espérait en créant ce service important [...] ont répondu à notre attente. Economie, célérité et bonne exécution dans les ordonnances, tout cela nous avons obtenu, grâce à la manière consciencieuse avec laquelle notre pharmacien, M. B. Raeber [sic] dirige notre pharmacie.» ${ }^{2}(1880$, p.12.) 1881 wird berichtet: «Le nombre des potions faites dans le courant de l'année a dépassé de beaucoup celui de l'année précédente. [...] Malgré cette manipulation considérable, aucune erreur, ni aucun accident n'ont eu lieu. Cela témoigne de l'activité et du savoir-faire de notre pharmacienchef. ${ }^{2}(1881$, p.12.)

Wie verhielt es sich nun mit den Reber vor Antritt der Stellung gemachten Zusagen, nachdem er seine fachliche Befähigung bewiesen und der Republik Genf namhafte Beträge eingespart hatte ? Die zuständige Persönlichkeit, mit der Reber seinerzeit verhandelt hatte, war inzwischen kaltgestellt worden, und niemand wollte für die Zusicherungen haften. Als die Offizin zu einer Staatsapotheke für die Versorgung sämtlicher öffentlicher Institutionen (der Polikliniken, der Gefängnisse, der Irrenanstalt usw.) erweitert werden sollte, wurde das Vorhaben vom Großen Rat abgelehnt. Da sich Reber in seiner Tätigkeit, die die Ellbogenfreiheit der Kliniken einschränkte, kaum besondere Sympathien in der Fakultät erwarb, war ihm eine akademische Laufbahn verbaut. Angesichts einer in jeder Hinsicht auch für die Zukunft unhaltbar werdenden Situation beschloß Reber nicht leichten Herzens, seine Stellung aufzugeben und eine eigene Apotheke am Boulevard James Fazy zu gründen. Ohne einen Hinweis auf den Rücktritt Rebers und eine Verdankung seiner Dienste meldet der Jahresbericht 1885 lakonisch: «Outre M. Bourget, pharmacien en chef, M. Narbel a été nommé pharmacien second.» Obgleich Gründung, Einrichtung und Führung einer Apotheke ganzen Einsatz verlangen, hatte Reber noch den Mut, sich wissenschaftlichen Neigungen und Arbeiten zu widmen. Gleichzeitig übernahm er als Mitgründer die Redaktion der monatlich zweimal erscheinenden, zweisprachigen Zeitschrift «Le Progrès - Der Fortschritt - Internationale Zeitschrift der Pharmazie und Therapie», für die er zahlreiche Beiträge schrieb. Angeregt durch die Empfehlungen des Internationalen Hygiene-Kongresses vom Jahre 1882 in Genf, war er nicht nur führend an der 
1887 erfolgten Gründung der «Société de crémation de Genève» beteiligt, sondern auch während rund dreier Jahrzehnte für die Gesellschaft tätig. Durch zahlreiche Vorträge und Schriften suchte er die Kremation ebenfalls in anderen Schweizerstädten und im Ausland zu fördern. 1888/89 wurde Reber vom Bundesrat als Mitglied der eidgenössischen Kommission für die Fachprüfung der Apotheker in Genf und als Mitglied zur Vorbereitung der dritten Auflage der «Pharmacopoea Helvetica » gewählt, für die er zahlreiche Abschnitte beisteuerte.

Nach fünf Jahren unermüdlicher und vielseitiger Tätigkeit erkrankte Reber an einer schweren Influenza. Ihre Folge war eine ernste Herzaffektion. Sie zwang ihn, seine Apotheke und seine Redaktionstätigkeit aufzugeben und die Zeitschrift eingehen zu lassen. Die Ärzte verordneten ihm einen längeren Aufenthalt in einer Höhe von über 1500 Metern, wozu Reber eine Ortschaft im Wallis aufsuchte. Die unfreiwillige Muße benützte er zum Ausbau seiner Sammlungen und zu prähistorischen und volkskundlichen Exkursionen in die Seiten- und Hochtäler des Kantons. Die wissenschaftlichen Ergebnisse veröffentlichte er in Fachschriften ${ }^{3}$ (p. 309 ff.).

\section{Die Sammlung Reber}

Seit seinem 20. Lebensjahr sammelte Reber alte Gegenstände, Bücher und Dokumente zur Geschichte der Pharmazie und später auch der Medizin. In jüngeren Jahren bedrückte ihn der Spott der Mitwelt über dieses Steckenpferd. Denn kaum jemand sah darin einen Sinn, «Gerümpel» zu sammeln und nicht mehr gebrauchte Gegenstände aus Apotheken und aus der medizinischen Praxis aufzuheben. Mancher dachte, er habe einen "Vogel im Kopf» (une arraignée au plafond), wie Reber bemerkt. Mit der ihm eigenen Energie verstand er es aber, die Bestände mit der Zeit zu äufnen, obgleich er sich Zurückhaltung für größere Ankäufe auferlegen mußte. "Il a fouillé partout, achetant ce qu'il voyait d'intéressant, au point de déséquilibrer son budget», schrieb später ein Fachkollege ${ }^{4}$ (p. 26).

Am 1. Mai 1893 waren 25 Jahre seit dem Eintritt Rebers in den pharmazeutischen Beruf vergangen. Dabei konnte er nicht nur auf ein erfolgreiches berufliches Wirken zurückblicken, sondern auch auf ein wahrhaft vielseitiges wissenschaftliches Schaffen, wie seine Gesamtbibliographie zeigt ${ }^{3}$. Zur Feier des Jubiläums vereinigten sich in- und ausländische Fachkollegen, um Reber eine Glückwunschadresse, eine goldene Medaille und ein Album mit Gratulationen zu überreichen. «Ils sont rares les hommes, qui, gagnant leur vie depuis la quinzième année de l'existence, travaillant et luttant continuellement, gardent les illusions idéales de leur jeunesse », bemerkt ein Zeitgenosse (1897 , p. XXXI). Gerührt von diesen Zeichen der Hochschätzung, beschloß Reber, das Ergebnis seiner gleichfalls ein Vierteljahrhundert währenden pharmazie- und medizinhistorischen Sammel- 
tätigkeit der öffentlichen Besichtigung zugänglich zu machen. Allerdings vergaß er auch erlittene Zurücksetzungen und Ärgernisse nicht, wenn er schreibt: "Pour me venger à ma façon de la mesquinerie, de la haineuse jalousie, de la petitesse et de la bassesse que j'avais depuis longtemps endurée, je me suis décidé d'organiser une exposition historique de médecine et pharmacie.» $\left(1909^{1}\right.$, p. 7.) Nach zeitraubenden, teils durch Krankheit verzögerten Vorbereitungen konnte Reber die Sammlung vom 26. Dezember 1893 bis 9. März 1894 im Genfer «Musée des Arts décoratifs » ausstellen. Leider fanden wir trotz vielen Bemühungen keinen Katalog. Immerhin ist es möglich, anhand der Aufzeichnungen Rebers eine kurze Übersicht zu geben, nämlich: «420 Apothekergefäße (aus Majolika, Fayence, Porzellan) aus z.T. berühmten Manufakturen des 15. bis 19. Jahrhunderts; 194. Apothekerflaschen aus Glas, mit farbigen, eingebrannten Aufschriften und Ornamenten; 37 Mörser (aus Bronze, Eisen, Marmor usw.); 140 Laborutensilien verschiedenster Art; eine Anzahl alte Waagen und feinziselierte Gewichtssätze; 85 alte pharmazeutische und medizinische Gegenstände (aus Assyrien, Ägypten, Palästina, Griechenland, Rom usw.); zahlreiche antike ärztliche Instrumente; Saugflaschen für Kinder und Kranke; 74 alte Reise- und Familienapotheken; etwa 800 alte Drogen und 110 antike Verpackungen für Medikamente; 11 Statuetten und über 500 Porträts und Medaillons im Zusammenhang mit Heilberufen; 196 ärztliche Rezepte aus dem 18. Jahrhundert; eine große Anzahl Diplome von Ärzten, Chirurgen, Apothekern seit dem 15. Jahrhundert; viele medizinische Manuskripte, darunter eines aus dem Ende des 13. oder Anfang des 14. Jahrhunderts, und schließlich eine Bibliothek von 800 Nummern, mit vielen Werken aus dem 16. und 17. Jahrhundert, davon zahlreiche gesuchte Unica.» (1909¹, p. 11-12.)

In über 50 in- und ausländischen Tageszeitungen sowie in Fachzeitschriften wurde über die Ausstellung berichtet. Eine hohe Ehre bedeutete es für Reber, daß sein ehemaliger Lehrer Friedrich August Flückiger (1828-1894), Ordinarius für Pharmazie in Straßburg, sich trotz seines Alters die Mühe nahm, nach Genf zu reisen. Aus seiner Feder stammt wohl die fachkundigste Würdigung der Ausstellung ${ }^{5}$. Reber selber hat über Flückigers Besuch berichtet: «Lui [Flückiger] s'étant laissé influencer par la médisance et la calomnie, - il me l'a avoué ensuite lui-même me disant en arrivant: 'Vous concevez qu'une exposition historique de médecine et pharmacie me force d'aller le voir. Je suis moralement obligé de le faire. Aussi je viens par pur acquit de conscience. Je pense en avoir fini en une heure et demie.' A l'entrée de l'exposition saisi par l'impression de l'ensemble et, après un instant de recueillement, il me disait: 'C'est toute autre chose qu'on voulait me faire croire, - on vous a beaucoup calomnié, M. Reber.' C'est la nuit qui le chassa de l'exposition. Le lendemain, dimanche, il y restait de nouveau 
depuis 8 heures du matin jusqu'à la nuit avec un court intervalle à midi. 'Et je suis encore bien loin d'en avoir fini' me disait-il, 'il faut que je reste encore demain.' La journée du lundi y passait encore. Il me déclarait qu'il quittait cette exposition à grand regret, son étude étant bien incomplète.» $\left(1909^{1}\right.$, p. 7 ff.)

Flückiger schreibt abschließend in seiner ausführlichen Würdigung: «Die Reber'sche Sammlung verläßt man mit aufrichtiger, dankbarer Bewunderung für die Hingebung, durch welche sie zu Stande gebracht worden ist, mit dem Wunsche, daß sie weiter vermehrt und namentlich, daß sie für alle Zeiten erhalten bleiben möge. In ersterer Hinsicht ist der Besitzer unausgesetzt und erfolgreich tätig, die Sicherheit der Sammlung ist aber in keiner Weise verbürgt. Die Stadt Genf ist so überreich an Sammlungen und vorzüglichen Bildungsmitteln aller Art, daß die Hoffnung berechtigt ist, dieses pharmazeutisch-medizinische Museum möchte jenen angereiht, d.h. öffentliches Eigentum werden. In den großen Herbarien de Candolle, Delessert und Boissier besitzt die Stadt mit den dazugehörigen Bibliotheken Hülfsmittel allerersten Ranges, vielleicht über eine Million sorgfältig eingelegter und geordneter Pflanzen. [...] Die Reber'sche Sammlung [...] dem übrigen wissenschaftlichen Apparate der unvergleichlichen Stadt beigefügt zu sehen, ist der Wunsch, zu dessen Begründung die vorstehenden Zeilen beitragen möchten. ${ }^{5}$ (p. 327.) Besonders weist Flückiger darauf hin, daß Reber zu den frühesten Begründern einer derartigen Sammlung gehöre, da H. Peters erst 1883 den Direktor des Germanischen Museums in Nürnberg veranlaßt habe, die Mitwirkung der ganzen Nation in Anspruch zu nehmen, um im Rahmen des Museums eine besondere, der Geschichte der Pharmazie im weitesten Sinne gewidmete Abteilung zu gründen. Reber bezeichnete später einmal seine Sammlung als «une collection d'un enthousiaste, à laquelle une vie entière a été consacrée» (190911, p. 4).

Einige Sammlungsgegenstände wurden auch an der Schweizerischen Landesausstellung in Genf 1896 gezeigt. 1898 reiste eine Kommission der Gesellschaft Deutscher Naturforscher und Ärzte nach Genf, um Reber zu ersuchen, ihr zur 70. Versammlung ihrer Gesellschaft vom 19. bis 24. September in Düsseldorf einen Teil seiner Sammlungsobjekte zur Ausstellung in den Räumen des dortigen Kunstgewerbemuseums zu überlassen. Reber erfüllte die Bitte, und die mit anderweitigen Leihgaben rund 4000 Nummern aufweisende «Ausstellung für Medizin und Naturwissenschaften», an deren Führungen sich auch Karl Sudhoff beteiligte, fand große Beachtung. Die Veranstalter des 1901 stattfindenden Ersten Russischen Ärzte-Kongresses wollten Rebers Kollektion in Moskau ausstellen. Da ihm dies zu gewagt erschien, schlug er als Ersatz die Zusendung eines Prachtsalbums mit 32 Großaufnahmen vor, was die Organisatoren guthießen. Sieben der Bilder wurden im Kongreßbericht wiedergegeben. 
1907 befaßte sich Reber aus uns unbekannten Gründen mit dem Gedanken an den Verkauf seiner Sammlung. Das beweist die Publikation eines Kataloges durch den Zürcher Antiquar H. Messikommer, in dem nicht nur die Sammlung, sondern auch Glasgemälde, Möbel usw. zum Verkauf angeboten wurden. Leider ist der in der Schweizerischen Landesbibliothek bibliographierte Katalog in den Beständen nicht mehr aufzufinden ${ }^{6}$. Es war nicht möglich, zuverlässig etwas über das Schicksal der ganzen Sammlung zu erfahren. Bekannt ist nur, daß Reber 1925 die Apothekergefäße der Universität Lausanne verkaufte. Es wurde ihm dafür eine jährliche Leibrente bewilligt, die ihm einen Lebensabend frei von Geldsorgen sichern sollte. Er konnte diesen Schutz nicht mehr genießen, da er bereits am 9. Juni 1926 starb. Vor 15 Jahren wurden diese Gefäße, wie uns die Herren Apotheker Dr. H. Käsermann und Dr. F. Ducommun mitteilten, an einer Tagung des Schweizerischen Apotheker-Vereins in Nyon ausgestellt. Mangels geeigneter Ausstellungsräume sind sie nun wieder im Schloß Nyon eingelagert. $\mathrm{Ob}$ auch noch ein Teil in Lausanne geblieben ist, scheint niemand genau zu wissen.

Reber war zeitlebens ein unermüdlicher Sammler. So legte er ein Herbarium an, das samt den ihm geschenkten Pflanzen rund 12000 Spezies zählte. Er stiftete es 1912 dem Botanischen Institut der Universität Genf. Seine Sammlung von über 2000 Mineralien und etwa 10000 Arten von Versteinerungen schenkte er dem Museum in Aarau, dem Seminar Wettingen und anderen Schulen. Ferner besaß er eine Sammlung von Bildern und Gemälden, Ex Libris und Objekten der Archäologie.

\section{Wissenschaftliche Arbeiten zur Geschichte der Medizin und Pharmazie}

Den Publikationen Rebers über diese Gebiete kann vor 1892 in einem weiteren Sinne nur das Buch «La crémation - Histoire, hygiène, technique» (1888) zugerechnet werden. Nach pharmaziehistorischen Beiträgen (1892 und 1894) trat Reber erstmals 1897 mit einem größeren Werk an die Öffentlichkeit. Er wollte nach Mustern des Auslandes, wo es Sammelwerke über hervorragende Ärzte und Naturforscher gab, Biographien bedeutender Pharmazeuten und Pharmakognosten zu einem Querschnitt durch das 19. Jahrhundert vereinigen (1897¹, p.XIII). Die Veröffentlichung verzögerte sich durch Krankheit und andere vom Willen des Herausgebers unabhängige Umstände. So erwähnt er, daß die Vorbereitung eine Korrespondenz von einigen tausend Briefen erfordert habe. Auch habe er sich mit Geduld wappnen müssen, da einzelne Briefwechsel und die $\mathrm{Zu}$ - und Rücksendung der Korrekturen, vor allem bei überseeischen Mitarbeitern, rund ein Jahr beansprucht habe (id., p. X). Der Inhalt des Buches besteht aus 105 Biographien. Einige stammen aus der Feder Rebers. 69 sind von Abbildung und Namenszug begleitet. Geschildert werden die wissenschaftlichen Leistungen und die be- 
rufliche Laufbahn von Pharmakognosten, die, falls sie nicht von der Botanik und Chemie herkamen oder Ärzte waren, vor der Aufnahme des Studiums noch eine praktische Apothekerlehre gemacht hatten. Das Werk wurde in 16 Lieferungen herausgegeben und fand in der Fachpresse günstige Aufnahme. Trotzdem blieb der Verkauf hinter den Erwartungen zurück, so daß Reber seinen ursprünglich weitergespannten Plan einschränken mußte. Er hatte das Buch im Selbstverlag veröffentlichen müssen und viel Zeit, Mühe und Geld dafür geopfert. Die Enttäuschung über den Mißerfolg und über die wenig idealistische Einstellung seiner Kollegen ist in seinen Worten nicht zu übersehen (id., p. IXff.). Aus den Jahren 1897-1899 sind einige größere Arbeiten zur Pharmaziegeschichte sowie kleinere medizinhistorische zu verzeichnen.

Nach mühevollen und jahrelangen Vorbereitungen erschien 1900 die Schrift «Balneologie und Climatotherapie. Versuch einer schweiz. Bibliographie der Litteratur auf den Gebieten des Badewesens, der Heilquellen, der climaterischen Kurorte», deren Redaktion Reber auf Ansuchen der «Centralkommission der Bibliographie für die schweizerische Landeskunde» übernommen hatte. Die Veröffentlichung ist das Ergebnis umfangreicher Erhebungen aus den Jahren 1893 und 1894 bei interessierten Kreisen unter Mitarbeit eidgenössischer und kantonaler Behörden und Verwaltungen sowie zahlreicher Fachwissenschaftler. Der erste allgemeine Teil ist der geschichtlichen Literatur gewidmet; der Rest gilt der alphabetischen und chronologischen Erfassung aller Publikationen nach Ortschaften, Bädern, Kurorten usw. «Als ich die Bearbeitung übernahm», schreibt Reber im Vorwort, «war ich weit entfernt, mir dieses Unternehmen so mühevoll und zeitraubend vorzustellen, wie es sich dann bei der Bearbeitung gezeigt hat», bei der er dann auch «nach und nach die Begeisterung verlor.» Angesichts der vielen Enttäuschungen und eingetretenen Schwierigkeiten war der Redaktor «trotz bestem Willen gezwungen, auf eine absolute Vollständigkeit zu verzichten». «Ich darf die Arbeit wenigstens mit dem Bewußtsein abgeben, mein Möglichstes getan zu haben », sagt er abschließend.

In den Jahren 1901-1903 finden wir nur wenige medizin- und pharmaziehistorische Arbeiten. Von 1905 an aber begann Reber mit der Beschreibung von Objekten seiner Sammlung. Ein Beitrag zur Kenntnis des Schießpulvers und der Geschütze (1908) fällt etwas aus dem Rahmen. Der Verfasser beschäftigte sich darin mit der Geschichte seines Namens und mit einem Namensvetter in Aarau, dessen Familienmitglieder während Jahrhunderten als Glocken- und Geschützgießer tätig waren.

In jenen Jahren war Reber - wie aus den Teilnehmerlisten hervorgeht - als Mitglied der Deutschen Gesellschaft für Geschichte der Medizin fast regelmäßig 
an ihren Jahresversammlungen, zum Teil auch als Referent, anwesend. Er kam dabei mit Karl Sudhoff zusammen, der seine Arbeiten wiederholt wohlwollend besprach. In einem Büchlein von 1909 vereinigte Reber die 1905 und 1906 im «Journal des Collectionneurs» erschienenen Berichte über die Gegenstände seiner Sammlung und fügte einige bisher ungedruckte Aufsätze bei, die wegen des Eingehens jener Zeitschrift nicht mehr hatten gedruckt werden können. Noch weitere acht Publikationen des Jahres 1909 sind der Geschichte der Medizin, Pharmazie, Chemie und Naturwissenschaften gewidmet und zeigen, wie Mannigfaltigem er seine Aufmerksamkeit zuwandte. Aus den folgenden Jahren ragen zwei Arbeiten zur Geschichte der Medizin in der Schweiz hervor $\left(1910^{5-6}\right)$; auch schrieb er weiter über seine Sammlung $\left(1910^{3-4}\right)$. Von 1911/12 sind nur drei Arbeiten zu nennen, darunter eine größere über Paracelsus, den er schon früher in Teilaspekten behandelt hatte. 1913 und 1914 stieg die Zahl der Veröffentlichungen wieder an; jedoch von 1915 an nahm die schriftstellerische Produktivität Rebers deutlich ab. $\mathrm{Ob}$ dafür die Kriegsjahre verantwortlich waren oder Alter und gesundheitliche Behinderungen, lassen wir offen. Rebers Gesundheitszustand hatte sich gefestigt, bis ihn 1904 eine rheumatische Erkrankung befiel. Nach ihren Erscheinungen und ihrem fortschreitenden Verlauf ist eine Polyarthritis anzunehmen. Sie nötigte Reber, seine Erwerbstätigkeit für kürzere oder längere Zeit einzuschränken oder zu unterbrechen, bis ihn schließlich die Krankheit ganz ans Bett fesselte und ihm eine schmerzensreiche Leidenszeit bereitete, von der ihn erst der Tod erlöste. Auch seine Arbeiten über archäologische und andere Themen, über die man sich nach der Aufzählung im «Catalogue $»^{3}$ ein Bild machen kann, fehlen von 1916 an. Nach 1920 konnten wir keine Veröffentlichungen mehr finden.

Die von Reber selber 1897, 1901, 1904 und 1908 herausgegebenen Verzeichnisse seiner Publikationen sind entweder nicht vollständig oder wegen ungenauer Zitierung mit Vorsicht zu benützen. Dies gilt auch für den von der Genfer Universität publizierten «Catalogue ${ }^{3}$, dessen Bearbeiter Rebers Angaben ungeprüft übernahm. Deshalb wurde versucht, hier erstmals eine vollständige Bibliographie seiner medizin- und pharmaziehistorischen Arbeiten möglichst aufgrund eigener Prüfung vorzulegen. Bei nur auswärts vorhandenen Zeitschriftenartikeln erhielten wir Auskünfte durch die Bibliotheken von Basel, Genf und Paris.

\section{Epilog}

Reber war erstaunlich vielseitig. Zeitnah erscheinen uns angesichts der heutigen Maßnahmen für den Schutz der Kulturgüter seine dahin zielenden Bemühungen. Bereits 1899 richtete er an die Behörden und die Öffentlichkeit einen eindringlichen Aufruf, worin er sich gegen die Zerstörung und die Geschäftemacherei (le 
vandalisme et l'affairisme) wandte. Die Publikation «De l'importance des monuments à sculpture préhistorique» ${ }^{11}$ fand zwar Beachtung in wissenschaftlichen Kreisen, weniger aber den gewünschten Erfolg bei den Behörden. Seine didaktischen Fähigkeiten bewies Reber während der Wintersemester 1900 und 1901, als er in der Société pédagogique in Genf ohne Honorar Deutschkurse (Grammatik, Konversation, Literatur) gab, an die sich die begeisterten Teilnehmer noch lange erinnerten. In den Jahren 1905/06 wirkte Reber während je einer Amtsdauer als Mitglied des «Grand Conseil» und als «Conseiller municipal» von Genf, wobei er sich nicht nur durch wohlvorbereitete Referate auszeichnete, sondern auch in insgesamt 26 Kommissionen beider Gremien tätig war. 1908 übernahm er das Amt eines Konservators des «Musée épigraphique»; fünf Jahre später wurde er zum Privatdozenten für dieses Fach an der Universität ernannt, eine Auszeichnung, die ihm wohl große Genugtuung bereitet hat. Bei seinem 60. Geburtstag wurde Reber im Hinblick auf seine Verdienste als « archéologue national» bezeichnet. Ein Zeitgenosse beschrieb ihn 1910 so: «Quand on songe un instant à l'incroyable activité de Reber, on reste véritablement confus. Il faut que chez lui la conception et l'exécution soient d'une égale rapidité. On croirait à peine, lorsque on le voit, cet homme toujours gai, aimable, avenant et souriant, toujours serviable, ayant pour chacun un mot de circonstance, n'ayant jamais l'air pressé, avançant pensivement, que sous cette calme apparence, un esprit travaille sans trève, sans relâche. ${ }^{8}$ (p. 70.) Auch sein medizinhistorischer Genfer Kollege H. Naegeli-Åkerblom hebt Rebers « unermüdlichen Fleiß» und seine Arbeitskraft hervor ${ }^{9}$. Von rund 30 wissenschaftlichen Akademien und Gesellschaften wurde er im Laufe der Jahre zum korrespondierenden oder Ehrenmitglied ernannt. Seine Begeisterungsfähigkeit beweist auch der Einsatz für eine - allerdings nicht verwirklichte - Eisenbahn ( Chemin de Fer de la Faucille»), deren Bau er als von großer Wichtigkeit für Genf bezeichnete ${ }^{1}$ (p. 44). Die Zivilcourage Rebers zeigt sein mutiges Eintreten im Prozeß Sezeguin, in dem er, von der Unschuld zweier verurteilter Franzosen überzeugt, sich nach eigenen eingehenden Erhebungen für eine Revision des Prozesses einsetzte und die Öffentlichkeit dafür zu gewinnen suchte (Akten in den Archives de l'Etat).

Reber hat keine Familie gegründet. Ersatz fand er in seinen wissenschaftlichen Neigungen, seinem öffentlichen Wirken und in den vielen freundschaftlichen und kollegialen Beziehungen im In- und Ausland. Auch mit dem Gründer der Zürcher Medizinhistorischen Sammlung, Gustav Adolf Wehrli, stand er im Verkehr. In einem Nachruf heißt es über Reber: «Le défunt n'était pas membre de la Société suisse de Pharmacie de laquelle il s'était séparé dans son jeune âge, à cause de l'incompatibilité de caractère qui l'avait toujours tenu à l'écart de ses collègues. 
Malgré ces circonstances fâcheuses, Reber a mérité l'admiration des pharmaciens suisses par son activité scientifique et ses capacités de chercheur et collectionneur. ${ }^{11}$ (Weitere Verdienste werden anschließend noch von dem nur mit einer Initiale zeichnenden Autor gewürdigt.) Ob hier auf die Tätigkeit Rebers im Kantonsspital angespielt wird, bei der wirtschaftliche Interessen der Apothekerschaft tangiert wurden, lassen wir offen. Jedenfalls war der Verstorbene zeit seines Lebens von hohen Idealen für den Apothekerberuf und von der Sorge für dessen Zukunft erfüllt, wie folgende Ausführungen zeigen: «Avant d'entrer en pharmacie déjà, je l'ai considérée comme le métier le plus libéral, le plus scientifique, et depuis que j'en fais partie, je n'ai cessé de me convaincre que réellement encore aujourd'hui elle pourrait l'être et elle devrait l'être. Hélas! l'égoïsme et la basse spéculation ont détourné beaucoup de disciples de ces belles maximes. Le résultat ne pouvait être douteux. La pharmacie a perdu son prestige scientifique.» $\left(1909^{1}\right.$, p. 6.)

Ein Nachruf sagt : «Sur beaucoup de ses travaux» - dies bezieht sich vor allem auf die archäologischen Arbeiten - «les savants ont sans doute quelques réserves à faire. Peut-être Reber manquait-il un peu de méthode et de sens critique. Il n'en a pas moins bien mérité [la reconnaissance] des disciples de Clio et laissera le souvenir d'un excellent homme, d'un travailleur infatigable, à l'esprit encyclopédique, curieux de toutes choses; d'un savant, original et prime-sautier dans ses propos, serviable et dévoué à la chose publique. ${ }^{10}$

Reber schrieb als 61 jähriger, was uns heute fast wie ein Lebensbekenntnis und Testament vorkommen will: «Ce que je retiens de cette grande et longue expérience de la vie est l'observation que ceux qui ne suivent pas les vieilles ornières de tout le monde, qui ne veulent rien savoir des coteries, qui au contraire se frayent de nouveaux chemins qu'ils entendent suivre librement et indépendamment, sont à la merci de toutes haines, de toutes les médisances et de toutes les calomnies. Il faut un caractère de fer pour lutter toute sa vie pour ses principes, vieillir et blanchir dans le chagrin et avoir encore le courage de continuer et de ne jamais rétrograder... J'ai eu enfin la sublime satisfaction de voir sanctionner mes efforts pour la fondation des musées au point de vue de l'histoire de la médecine et de la pharmacie par le monde scientifique tout entier. Aujourd'hui on m'envie le résultat et on me félicite de mon succès. C'est un peu tard.» $\left(1909^{1}\right.$, p. 14.) 


\title{
I. Ungedruckte Quellen
}

\author{
Archives de l'Etat, Genève
}

Autographes G-Z. Mss. hist. 254.

Documents relatifs à la Famille Reber. Cote: 1295, 1908.

Bibliothèque publique et universitaire, Genève

B. Reber, Opuscules, t. I, II, IV, VI, IX, X (6 vols., 1871-1897). Cote: V 28/63 und Na 984.

Opuscules sur l'histoire de médecine.

\section{Gedruckte Quellen}

\section{Sekundärliteratur}

1) A propos de l'anniversaire de Burkhard Reber (ohne Verfasserangabe). Tirés à part du journal Le Genevois, Genève (Imprimerie Centrale) 1918, 47 p. (Dabei handelt es sich nach unserem Vergleich mit dem Original nur teilweise um einen Sonderdruck. Ein wesentlicher Teil des Textes ist in der Zeitung nicht enthalten und wurde nur in der Broschüre gedruckt. Offen bleibt der Name des Verfassers. Nach Text und Stil dürfte Reber selbst Material zur Verfügung gestellt haben.)

2) Rapports de la Commission administrative de l'Hôpital cantonal de Genève, 18781886.

3) Catalogue des ouvrages, articles et mémoires publiées par les Professeurs, Privatdocents de l'Université de Genève, rassemblés par Albert Kohler. Documents pour servir à l'histoire de l'Université de Genève, Vol. VI, Genève 1916, p. 309-327. (Diese Bibliographie enthält 303 Publikationen Rebers aus den verschiedensten Gebieten, ist aber nicht vollständig.)

4) Ducommun, F.E., Pharmaciens d'autrefois. Notice publiée par la Société genevoise de Pharmacie à l'occasion du quatrième Centenaire de l'Université de Genève.

5) Flückiger, F.A., Die historische pharmaceutisch-medicinische Sammlung des Apothekers Burkhard Reber in Genf. Apotheker-Zeitung 2 (Berlin 1894), p.289-293, 297-300, 305-307, 315-317, 325-327.

6) Katalog hervorragender Sammlungsstücke. Sammlung von Glasgemälden des 13. bis 15. Jahrhunderts, Öfen, Möbel etc. Das B. Reber'sche Medizinisch-pharmaceutische Museum in Genf. Zum freihändigen Verkauf angeboten durch Heinrich Messikommer, zur Meise, Zürich (1907); illustriert, IV + 51 p.

7) Mitteilungen zur Geschichte der Medizin und der Naturwissenschaften 1 (1903), p. 242, 244; 4 (1909), p. 421.

8) Carnoy, Henry, Artikel über B. Reber in: Dictionnaire biographique international des écrivains, des artistes, des sociétés savantes, des collectionneurs, t.VIII, Paris 1910, p. 69-71.

9) Mitteilungen zur Geschichte der Medizin und der Naturwissenschaften 5 (1906), p. 523. (Hans Naegeli-Åkerblom [1868-1911], Privatdozent für Medizingeschichte in Genf, war mit Reber befreundet, starb jedoch im besten Mannesalter.) 
10) Journal de Genève, $47^{\mathrm{e}}$ année, 12 juin 1926, p.6.

11) Schweiz. Apotheker-Zeitung 64 (1926), p. 300.

12) De l'importance des monuments à sculpture préhistoriques. Appel aux autorités, aux propriétaires de monuments, et à tous les amis de l'histoire nationale, pour la conservation de ces monuments. Sonderdruck aus: Bulletin de l'Institut national genevois, t. XXXV (1899), $52 \mathrm{p}$.

Der Universitätsbibliothek Basel, der Bibliothèque publique et universitaire, Genève, und der Bibliothèque de la Faculté de médecine, Paris, danken wir für Überprüfungen und Ergänzungen in der Bibliographie Rebers. Das Bild Rebers verdanken wir der Bibliothèque publique et universitaire de Genève, und seine Unterschrift haben wir einer Widmung im Vorsatzblatt des Buches $1897^{1}$ entnommen.

\section{Bibliographie der pharmazie- und medizinhistorischen Schriften Burkhard Rebers}

1888 1 La crémation - Histoire, hygiène, technique. Genève (R. Burckhard) 1888, 70 p., ill.

$1892^{1}$ Une médaille pharmaceutique. Bull. Soc. suisse de numismatique 11 (1892), p.14-15.

18941 Eine Basler Apotheker-Taxe vom Jahre 1647. Pharm. Post 27 (Wien 1894), p. 105108.

$1894^{2}$ Pharmaceutisches aus dem Elsaß anfangs des 17. Jahrhunderts. Pharm. Post 27 (Wien 1894), p.285-290.

$1894^{3} \quad$ Nachruf an Flückiger. Pharm. Post 27 (Wien 1894), p.611-614.

$1897^{1}$ Gallerie hervorragender Therapeutiker und Pharmakognosten der Gegenwart Galerie d'éminents thérapeutistes et pharmacognostes contemporains. Genève (Imprimerie Paul Dubois) 1897. 408 p., ill.

$1897^{2} \quad$ L'habit des médecins pendant la peste. Janus 1 (Amsterdam 1897), p. 297-300.

$1897^{3} \quad$ Notice bibliographique sur le prof. Planchon. Janus 2 (Amsterdam 1897), p.81-85.

$1897^{4}$ Schweizerische Beiträge zur Geschichte der Pharmacie.

I. Einleitung und Biographisches über den Apotheker und Staatskanzler Renward Cysat, Luzern. Schweiz. Wschr. f. Chemie und Pharmacie 35 (1897), p.518-522, 535-539.

II. Apotheken-Visitationen von 1588 und 1592 nebst der Apotheker-Ordnung vom Jahr 1592. Id., p.548, 574-576, 592-595, 611-613.

III. Die sogenannte Reform der Apotheken in Genf und der Proceß des Apothekers Joseph Forer. Id. 36 (1898), p.16-19, 33-36, 46-48, 82-85, 92-94, 113-117, 125-127, 136-138, 142-148.

Diese Arbeiten wurden als Sonderdruck unter dem Titel Schweiz. Beiträge zur Geschichte der Pharmacie, Zürich (Orell Füßli) 1898, 50 p., veröffentlicht.

$1898^{1}$ L'habit des médecins pendant la peste. Revue méd. suisse romande 18 (1898), p.730-733.

$1898^{2}$ Beiträge zur Geschichte der Pharmacie. Pharm. Post 31 (Wien 1898), p.413-415, 438-441, 473-475, 534-536, 545-547, 573-575, 598-600, 611-612, und 32 (1898), p.259-267, 271-278, 295-297. 
Diese Beiträge wurden als Sonderdruck Beiträge zur Geschichte der Pharmacie, Wien (Verlag der Wochenschrift der Pharmaceutischen Post) 1899, veröffentlicht.

18991 Erlebnisse eines jungen Arztes. Sonntagsblatt des Bund (Bern 1899), p.4-6, 11-14, 20-22, 28-31, 36-38.

1899² Römische Anzeichen der Ausübung von Gesundheitspflege und Medicin in Baden (Schweiz). Janus 4 (Amsterdam 1899), p.399-405.

$1899^{3}$ Der Safran in der Geschichte. Pharm. Rundschau 25 (Wien 1899), p.841-843.

18994 Nachdruck in: Correspondenz-Blatt für Schweizer Aerzte 30 (1900), p.785-787.

$1899^{5}$ Betrachtungen über die Pest. Pharm. Post 32 (Wien 1899), p.563-565, 627-628, 643-649, und 33 (1900), p.61-65.

$1900^{1}$ Balneologie und Climatotherapie. Versuch einer schweiz. Bibliographie der Litteratur auf den Gebieten des Badewesens, der Heilquellen, der climaterischen Kurorte. - Balnéologie et Climatothérapie. Essai d'une bibliographie suisse sur la littérature balnéologique. Bibliographie der Schweiz. Landeskunde - Bibliographie nationale suisse, Fasc.IV 3, Bern (K.J. Wyß) 1900, 111 p.

$1900^{2}$ Verordnung gegen Kurpfuscher und unerlaubte Arzneibücher am Ende des XVI. Jahrhunderts. Pharm. Post 33 (Wien 1900), p.302-305.

$1900^{3}$ Pharmaceutische Skizzen aus Paris. Pharm. Post 33 (Wien 1900), p.332-335, 465-469, 501-507.

$1900^{4}$ Ein Beitrag zur Geschichte der Syphilis. Correspondenz-Blatt für Schweizer Aerzte 30 (1900), p.501-503.

$1900^{5}$ Vorsichtsmaßregeln gegen die Pest in früheren Jahrhunderten. Id., p.686-691, 719-724.

1901 Eine Basler Apotheker-Ordnung vom Jahre 1701. Pharm. Post 34 (Wien 1901), p. 201-203.

$1901^{2}$ Pestverordnungen für die Schulen und den gemeinen Mann aus dem Ende des 16. Jahrhunderts. Schweiz. Arch.f. Volkskunde 5 (1901), p.302-305.

$1901^{3} \quad$ Ein Beitrag zur Geschichte der Ruhrepidemie. Correspondenz-Blatt für Schweizer Aerzte 31 (1901), p.353-356, 385-387, 433-435.

1901 Der Berufseid der Ärzte und Scherer im XVI. Jahrhundert. Id., p.571-572.

$1901^{5}$ Die Zürcher Apotheker-Taxe vom Jahr 1577. 14 p. Originalarbeit in: Burkhard Reber, Beiträge zur Geschichte der Medicin und der Pharmacie, II. Serie, Genf 1901.

$1902^{1}$ Die Volksmedizin der Augenkrankheiten. Pharm. Post 35 (Wien 1902), p.2-7, 19-21.

Die Arbeiten $1899^{5}, 1900^{2-3}, 1901^{1-2}, 1902^{1}$ wurden als Sonderdruck unter dem Titel Beiträge zur Geschichte der Medecin und der Pharmacie, I. Serie, Wien (Druck von Ferdinand Brück \& Söhne) 1900, 72 p., veröffentlicht.

Die Arbeiten $1899^{1}, 1900^{4-5}, 1901^{2-5}$ wurden als Sonderdruck Beiträge zur Geschichte der Medicin und der Pharmacie, II. Serie, Genf (Buchdruckerei Eggimann \& Cie.) 1901, veröffentlicht.

1903 Pharmacie de poche d'un médecin romain. Bull. Soc. française d'hist. méd. 2 (1903), p.369-375. 
1905 Historie de la médecine, de la pharmacie et des sciences naturelles. J. des Collectionneurs 1 (1905), p.129-134.

$1905 / 06^{1}$ Vases pharmaceutiques en faïence et majoliques italiennes. Id., p.165-168, 181184.

1905/06² Poteries pharmaceutiques de France, Belgique, Allemagne, Autriche etc. Id., p.221-222.

$1905 / 06^{3}$ La poterie suisse. Id., p.234-237.

1905/064 Suite des faïences. Flacons de pharmacie en verre et récipients en bois. Id., p.246-247.

1905/065 La thériaque. Id., p.247-250.

1905/06 Mortiers et appareils de laboratoire. Id., p.261-264.

1905/06 ${ }^{7}$ Les antiquités genevoises de ma collection et les anciennes pharmacies de Genève. Id., p.274-276.

1905/06 ${ }^{8}$ Les épidémies, particulièrement la peste, dans l'ancien temps. Id., p.291-294.

$1906^{1}$ De la valeur de l'histoire d'une science. J. des Collectionneurs 3 (1906/07), p.422-424.

$1906^{2}$ Une pharmacie portative d'un médecin romain. Id., p.424.

$1906^{3}$ La vente des poisons à Genève au XIX ${ }^{\mathrm{e}}$ siècle. Id., p.388-389.

$1906^{4}$ Les laboratoires des anciens temps. Id., p.375-378.

$1906^{5}$ L'histoire de la médecine dans les tendances actuelles. Id., p.350-353.

$1906^{6} \quad$ L'apprentissage en médecine, pharmacie et chirurgie. Id., p.317-318.

$1906^{7}$ Considérations sur la crémation, particulièrement au point de vue de ses rapports avec la médecine légale. Bull. Soc. de Crémation de Genève VI (1906), p.3-40.

$1906^{8}$ Zwei neue Dokumente über Leonhard Thurneysser zum Thurn. Mitteilungen zur Geschichte der Medizin und der Naturwissenschaften 5 (1906), p.431-439.

$1906^{9}$ [Le philosophe Eduard de] Hartmann, ${ }^{*}$ 23. II.1842. Le Genevois, $\mathrm{N}^{0} 154 \mathrm{du}$ 3 juillet 1906.

$1906^{10}$ Le chirurgien Fabricius Hildanus. Id., $N^{\circ} 249$ du 23 octobre 1906.

$1907^{1}$ Ein Lobgedicht des Fabricius Hildanus auf den «Wasserschatz» des Tabernaemontanus, sowie Anweisungen des Gebrauches der Bäder von Baden im Aargau und von Markgrafen-Baden. Mediz. Klinik 3 (Berlin 1907), p.148-150.

$1907^{2}$ Quelques appréciations sur Theophrastus Paracelsus. Bull. Soc. française d'hist. méd. 6 (1907), p.90-100.

$1907^{3} \quad$ Ein Brief Karl Friedrich Mohr's. Pharm. Post 40 (Wien 1907), p.409-410.

$1907^{4}$ Ein Beitrag zur Kenntnis der freundschaftlichen Beziehungen Liebigs. Mitteilungen zur Geschichte der Medizin und der Naturwissenschaften 6 (1907), p. 497-502.

$1907^{5}$ Friedrich Schlagdenhauffen. Ein kurzes Lebensbild. Pharm. Post 40 (Wien 1907), p.639-645.

1908 $\quad$ Nouveaux documents sur la peste. La France médicale 55 (1908), p.41-43.

$1908^{2}$ Ein Wort über Paracelsus. Als Vorbemerkung für eine ausgedehntere Studie. Pharm. Post 41 (Wien 1908), p.621-629.

$1908^{3} \quad$ Sir John Evans. Le Genevois, N ${ }^{\circ} 136$ du 12 juin 1908.

$1908^{4}$ Ein Beitrag zur Kenntnis des Schießpulvers und der Geschütze. Mitteilungen zur Geschichte der Medizin und der Naturwissenschaften 7 (1908), p.427-429. 
$1909^{1}$ Considérations sur ma collection d'antiquités au point de vue de l'histoire de la médecine, la pharmacie et les sciences naturelles. Genève (Imprimerie L. Jarry's \& fils) 1909, 154 p. (Dieses Buch ist ein Sonderdruck der Arbeiten 1905/06 ${ }^{1-8}, 1906^{1-6}$, enthält aber wegen Eingehens des Journal des Collectionneurs noch folgende unpublizierte Arbeiten: Intérieurs d'alchimistes, p.119-123; Les portraits, p.124-129; La caricature en médecine et en pharmacie, p.130-133; Le charlatanisme et la réclame, p.134-135; La peinture sacrée en médecine, p.136-137; Numismatique médicale et pharmaceutique, p.138-139; Principales publications parues jusqu'à ce jour sur mon Musée médico-pharmaceutique, p.140-144; Dernier mot, p.145146.)

1909² Geschichtliche Forschungen über die Pest. Der Bund (Bern), Nr.169 vom 11.April 1909 , p.5.

$1909^{3}$ Zum hundertsten Geburtstag Charles Darwins. Mitteilungen zur Geschichte der Medizin und der Naturwissenschaften 8 (1909), p.249-254.

$1909^{4}$ Deux documents inédits de Théodore Tronchin. Bull. Soc. française d'hist. méd. 8 (1909), p.356-365.

$1909^{5}$ Le chirurgien Fabrice de Hilden. La France médicale 56 (1909), p.317-319.

$1909^{6}$ Erinnerungen an Julius Trapp. Pharm. Post 42 (Wien 1909), p.77-87.

19097 Bilder aus der Geschichte der Chemie. Id., p.517-523.

19098 Die Standgefäße der alten Apotheken. Id., p.893-901.

$1909^{9}$ Un document inédit de Lamarck. La France médicale 56 (1909), p.408.

$1910^{1}$ Standgefäße alter Apotheken aus Österreich-Ungarn und Spanien. Pharm. Post 43 (Wien 1910), p.69-75, 709-714.

$1910^{2}$ Une lettre inédite de Pierre Bayen, suivie de quelques observations. Bull. Soc. française d'hist. méd. 9 (1910), p.50-63.

$1910^{3}$ Einzelheiten aus dem Innern der ehemaligen Apotheken und Laboratorien. Pharm. Post 43 (Wien 1910), p.509-516.

$1910^{4}$ Standgefäße alter Apotheken: Fayencen der übrigen Länder Europas. Id., p.709714.

$1910^{5} \quad$ Einige weitere Beiträge zur Geschichtsforschung über die Pest. Mitteilungen zur Geschichte der Medizin und der Naturwissenschaften 9 (1910), p.343-359.

$1910^{6}$ Zwei schweizerische Geburtsstühle. Gynaecologia Helvetica 10 (1910), p.415-420.

$1911^{1}$ Quelques appréciations de ces derniers temps sur Paracelse. "Bibliothèque historique de la France médicale», Paris 1911, 30 p.

19112 Résumé dans La France médicale 58 (1911), p.303-309.

$1912^{1}$ Lettres inédites des célèbres médecins Tissot et Zimmermann. La France médicale 59 (1912), p.281-285.

$1913^{1}$ Le docteur [Jean-François] Coindet. - L'emploi de l'iode contre le goitre. - Les crétins du Valais. Aesculape 3 (Paris 1913), p.93-96.

19132 Quelques objets du Musée Reber. Bull. Soc. d'hist. pharm. 1 (Paris 1913), p.17-20.

$1913^{3}$ Une note de médecin remontant à deux siècles. La France médicale 60 (1913), p.302-303.

19141 Diplôme d'un barbier-chirurgien. La France médicale 61 (1914), p.96-97. 
$1914^{2}$ Une consultation du docteur [Théodore] Tronchin. Bull. Soc. française d'hist. méd. 13 (1914), p.102-105.

$1914^{3}$ Mesures prophylactiques contre la rage à la fin du XVIII ${ }^{\mathrm{e}}$ siècle. Id., p.186-191.

1914 Quelques remarques sur la Thériaque, le Mithridate, l'Opiat de Salomon et l'Orviétan. Id., p.460-478.

$1914^{5} \quad$ Contre les fièvres chaudes. La France médicale 61 (1914), p.97.

$1914^{6}$ Populäre Behandlung der Frauenkrankheiten im 15. Jahrhundert. Gynaecologia Helvetica 14 (1914), p.369-378.

19147 Lettres entre deux célèbres pharmaciens de Genève, Henri Albert Gosse et PierreFrançois Tingry, Genève 1914, 19 p.

$1915^{1}$ Une notice sur la vie scientifique à Genève à l'époque de la Restauration. $L a$ Tribune de Genève 37, $\mathrm{N}^{\mathrm{o}}$ du 28 février et du $1^{\mathrm{er}}$ mars 1915.

$1917^{1}$ Les grands pharmaciens: V.François Tingry (1743-1821). Quelques notes sur sa vie et sur sa découverte du Sel Seydlitz en France. Bull. Soc. hist. pharm. 1 (Paris 1917), p.313-319.

$1918^{1}$ Un chapitre de physiologie végétale au XVIII ${ }^{\mathrm{e}}$ siècle. Id., 1 (1918), p.388-392.

1918 ${ }^{2}$ Notes sur Tingry: Une démarche du premier Comité de la Société de Pharmacie de Lyon en 1807. Bull. Soc. Pharmacol. 25 (1918), p.111-117.

$1920^{1}$ Faïence et majoliques. Publié sous les auspices de «Pages d'art». Genève (Editions «Sonor ») 1920, IV + 40 p., ill.

\section{Summary}

Burkhard Reber (1848-1926) was a Swiss farmer's son. In 1879 he became chief pharmacist of the cantonal hospital of Geneva. He was a great collector of old objects, books and documents of the history of pharmacy and medicine. Today, a part of his collection is stocked up in the castle of Nyon. Reber was also a busy writer in the field of the history of pharmacy. A bibliography of his works on the subject is given.

Paul Röthlisberger

Thunstraße 44

3005 Bern 